\title{
Motor unit number index detects the effectiveness of surgical treatment in improving distal motor neuron loss in patients with incomplete cervical spinal cord injury
}

Jun $\mathrm{Li}^{1,2+}$, Yancheng Zhu ${ }^{3+}$, Yang $\mathrm{Li}^{2}$, Shisheng $\mathrm{He}^{1^{*}}$ and Deguo Wang ${ }^{2^{*}}$

\begin{abstract}
Background: Recovery of motor dysfunction is important for patients with incomplete cervical spinal cord injury (SCI). To enhance the recovery of muscle strength, both research and treatments mainly focus on injury of upper motor neurons at the direct injury site. However, accumulating evidences have suggested that $\mathrm{SCl}$ has a downstream effect on the peripheral nervous system, which may contribute to the poor improvement of the muscle strength after operation. The aim of this study is to investigate the impact of early vs. delayed surgical intervention on the lower motor neurons (LMNs) distal to the injury site in patients with incomplete cervical SCl.

Methods: Motor unit number index (MUNIX) was performed on the tibialis anterior (TA), extensor digitorum brevis (EDB) and abductor hallucis (AH) in 47 patients with incomplete cervical SCl (early vs. delayed surgical-treatment: 17 vs. 30) and 34 healthy subjects approximately 12 months after operation. All patients were further assessed by American spinal injury association (ASIA) motor scales and Medical Research Council (MRC) scales.

Results: There are no difference of both ASIA motor scores and MRC scales between the patients who accepted early and delayed surgical treatment $(P>0.05)$. In contrast, the patients undergoing early surgical treatment showed lower MUSIX values in both bilateral EDB and bilateral TA, along with greater MUNIX values in both right-side EDB and right-side TA, compared to the patients who accepted delayed surgical treatment $(P<0.05)$.

Conclusions: Cervical SCI has a negative effect on the LMNs distal to the injury site. Early surgical intervention in Cervical SCl patients may improve the dysfunction of LMNs distal to the injury site, reducing secondary motor neuron loss, and eventually improving clinical prognosis.
\end{abstract}

Keywords: Spinal cord injury, Motor unit number index, Motor unit loss, Optimal timing for surgery, Trans-synaptic degeneration

\footnotetext{
* Correspondence: heshisheng0321@126.com; wangdeguo0321@126.com

†Jun Li and Yancheng Zhu contributed equally to this work.

'Department of Orthopedics, College of Clinical Medicine, Shanghai Ten

Hospitals of Nanjing Medical University, 301 Yanchang Middle Road, Jing'an

District, Shanghai 200072, China

Department of Orthopedics, Shanghai Songjiang District Central Hospital,

Shanghai 201600, China

Full list of author information is available at the end of the article
}

C C The Author(s). 2020 Open Access This article is licensed under a Creative Commons Attribution 4.0 International License, which permits use, sharing, adaptation, distribution and reproduction in any medium or format, as long as you give appropriate credit to the original author(s) and the source, provide a link to the Creative Commons licence, and indicate if changes were made. The images or other third party material in this article are included in the article's Creative Commons licence, unless indicated otherwise in a credit line to the material. If material is not included in the article's Creative Commons licence and your intended use is not permitted by statutory regulation or exceeds the permitted use, you will need to obtain permission directly from the copyright holder. To view a copy of this licence, visit http://creativecommons.org/licenses/by/4.0/. The Creative Commons Public Domain Dedication waiver (http://creativecommons.org/publicdomain/zero/1.0/) applies to the data made available in this article, unless otherwise stated in a credit line to the data. 


\section{Background}

Recovery of motor dysfunction is important for patients with incomplete cervical spinal cord injury (SCI) since it is essential for improving health-related quality of life [1]. To enhance the recovery of muscle strength, both research and treatments mainly focus on both primary and secondary injury of upper motor neurons (UMN) at the direct injury site $[2,3]$. In many different neuroprotective treatments, surgical decompression is demonstrated as one of the most important methods, and accumulating evidence has suggested that early operation can relieve both mechanical compression and microcirculation disturbance of cervical cord, therefore improving the clinical prognosis $[3,4]$.

The pathophysiology of cervical SCI is complex, and recently published studies revealed obvious electrophysiological abnormalities in distal paralyzed muscles in patients with SCI [1, 5-7], suggesting the degeneration of both spinal motoneurons and peripheral motor axons in regions caudal to the level of direct injury, which may contribute to the poor improvement of the muscle strength. Unfortunately, few studies have involved the effects of early surgical treatment on the loss/ dysfunction of distal motor neurons, although this issue is important for clinician to establish suitable treatment of cervical SCI.

Motor unit number index (MUNIX) is a recently developed quantitative method that provides an estimated index of the number and size of the functional motor units in the tested muscle [8]. According to the previous studies, MUNIX was demonstrated to be very sensitive in detecting motor unit loss in many different neuromuscular diseases [8-11], and both Li et al. and Marciniak et al. demonstrated MUNIX detection can be used to assess the integrity of lower motor neuron in patients with SCI $[12,13]$.

The aim of the present study is to quantify the functional motor units in the lower limb muscles in patients with incomplete cervical SCI who accepted early $(\leq 72 \mathrm{~h})$ versus delayed $(>72 \mathrm{~h})$ surgical treatment.

\section{Methods}

\section{Subjects}

A total of 47 patients with incomplete cervical SCI and 34 healthy subjects were analyzed retrospectively in this study. In the present study, seventeen patients with cervical SCI underwent early surgical treatment $(\leq 72 \mathrm{~h})$, and the other 30 patients underwent delayed surgical treatment $(>72 \mathrm{~h})$ (Table 1). All subjects in cervical SCI patient group were recruited in SongJiang Hospital from December 2015 to June 2017. The study protocol was approved by Human Ethics Committees (Shanghai Songjiang District Central Hospital, KYLL2015-263). All subjects gave informed consent.

The subjects in normal control group were chosen based on the inclusion and exclusion criteria published

Table 1 Characteristics of patients with cervical SCl in both surgical treatment groups

\begin{tabular}{|c|c|c|}
\hline & Early surgical treatment group & Delayed surgical treatment group \\
\hline Number of subjects & 17 & 30 \\
\hline Age range (years) & $45.0 \pm 12.2$ & $47.4 \pm 13.1$ \\
\hline Height range $(\mathrm{cm})$ & $164.7 \pm 8.9$ & $165.9 \pm 9.2$ \\
\hline Gender (Male vs. Female) & 12 vs. 5 & 24 vs. 6 \\
\hline Time from injury to surgery (days) & $1.9 \pm 0.7$ & $17.2 \pm 7.9$ \\
\hline \multicolumn{3}{|l|}{ Severity of SCl } \\
\hline ASIA B & $2 / 17(11.8 \%)$ & $2 / 30(6.7 \%)$ \\
\hline ASIA C & 10/17 (58.8\%) & $13 / 30(43.3 \%)$ \\
\hline ASIA D & $5 / 17(29.4 \%)$ & $15 / 30(50 \%)$ \\
\hline \multicolumn{3}{|c|}{ Imaging abnormalities (n/total patient (\%)) } \\
\hline Cervical fracture & 5/17 (29.4\%) & $8 / 30(26.7 \%)$ \\
\hline Intramedullary high-signal lesion & $7 / 17(41.2 \%)$ & $18 / 30(60.0 \%)$ \\
\hline \multicolumn{3}{|c|}{ Mechanism of injury (n/total patient (\%)) } \\
\hline Falls & 6/17 (35.3\%) & $9 / 30(30.0 \%)$ \\
\hline Vehicle accidents & $11 / 17(64.7 \%)$ & $21 / 30(70.0 \%)$ \\
\hline \multicolumn{3}{|l|}{ Surgical approach (n/total patient (\%)) } \\
\hline Anterior & 5/17 (29.4\%) & $7 / 30(23.3 \%)$ \\
\hline Posterior & 13/17 (76.5\%) & 20/30 (66.7\%) \\
\hline Combined & 1/17 (5.9\%) & $3 / 30$ (10.0\%) \\
\hline
\end{tabular}

SCI Spinal cord injury, ASIA ASIA (American Spinal Injury Association) impairment scale 
previously [5]. The inclusion criteria for cervical SCI patients includes (1) a clear history of trauma; (2) different degrees of sensory and motor impairments in both upper limbs and/or the lower limbs with a variable effect on bladder function; (3) magnetic resonance imaging (MRI) and/or Computed tomography (CT) demonstrating SCI at the cervical segment without the injuries in the level of thoracic and lumbar spine injuries. The exclusion criteria for cervical SCI patients includes previous spinal surgery, polyneuropathies, radiculopathies, plexopathies, focal neuropathies, muscle disorders, diabetes, diseases of the central nervous system, syringomyelia, spinal cord tumour/inflammation/infection, spinal deformities and severe degenerative diseases of thoracic and lumbar segments.

\section{Testing methods}

\section{Motor unit number index}

The MUNIX detection was applied in both 47 patients with incomplete cervical SCI approximately 1 year after operation and 34 healthy subjects. The MUNIX method described by Nandedkar et al. was used in this study [8]. The maximal compound muscle action potential (CMAP) was recorded bilaterally from the tibialis anterior (TA), extensor digitorum brevis (EDB) and abductor hallucis (AH) in a belly-tendon montage (filters: $3 \mathrm{~Hz}-10$ $\mathrm{kHz}$ ) to supramaximal stimulation. Subsequently, surface interference pattern (SIP) for ten different force levels of isometric contraction was recorded in a $300-\mathrm{ms}$ window (filters: $10 \mathrm{~Hz}-1000 \mathrm{~Hz}$ ). According to these measurements, both MUNIX values and motor unit size index (MUSIX) values for these three muscles were measured.

For the evaluation of the reproducibility, left-side MUNIX measurements of 15 healthy subjects and 21 patients with cervical SCI were tested twice by the same examiner. The intervals between these 2 tests were longer than $60 \mathrm{~min}$, and the electrodes were completely removed after the initial test.

All electrophysiological examinations were carried out by Keypoint EMG machine (Medtronic Dantec, Skovlunde, Denmark) with a skin temperature $>32^{\circ} \mathrm{C}$. MUNIX values cannot be measured when the following conditions occur: SIP area $<20 \mathrm{mV}$.ms, ideal case motor unit count $($ ICMUC) $>100$, SIP area/CMAP area $<1$, or CMAP amplitude $<0.5 \mathrm{mV}$.

\section{Clinical function examination}

All 47 patients with cervical SCI accepted American spinal injury association (ASIA) classification to identify the severity of SCI at the time of admission. All of these patients further underwent muscle strength examination in all tested muscles graded by the Medical Research Council (MRC) scales and American spinal injury association (ASIA) motor scores approximately 1 year after operation.

\section{Statistical methods}

The measurements were analyzed by SPSS 18.0 (IBM, Armonk, NY), and Medsci simple size tools (Shanghai, China) were used to calculate sample size. The measurements among healthy subjects and different cervical SCI patient groups were tested using one-way ANOVA (least significant difference correction). The preoperative and postoperative ASIA motor scores were compared by the paired t-test.

Pearson or Spearman correlation coefficient analysis (CCA) was used to evaluate the relationship between MUNIX values and MRC scales in each patient group. The test-retest reproducibility of MUNIX in healthy subjects and patients with cervical SCI was analyzed using interclass correlation coefficient (ICC) methods.

A $P$-value less than 0.05 was considered significant.

\section{Results}

A significantly positive correlation of all MUNIX values between the initial and second tests was demonstrated in all tested muscles in both normal control and cervical SCI patient groups $(P<0.05$, Fig. 1$)$, and good reproducibility of all MUNIX measurements was further confirmed by ICC in both subject groups (Table 2). Furthermore, there is an obvious positive correlation between the MUNIX values and MRC scales in all tested muscles in both patient groups $(P<0.05$, Fig. 2$)$.

In the delayed surgical treatment group, the MUNIX values were not recorded from all tested muscles in two patients. Furthermore, absent MUNIX values were also observed in bilateral TA in one patient and in unilateral EDB in two patients. In addition, the other two patients in this group presented with absent MUNIX values in bilateral EDB, along with absent MUNIX values in leftside TA in one of these 2 patients. Therefore, seven (7/ 30, 23.3\%) patients who accepted delayed surgical treatment presented with absent postoperative MUNIX values in at least one of the tested muscles in this study. In contrast, only one $(1 / 17,5.9 \%)$ patient in the early surgical treatment group presented with absent MUNIX values in bilateral EDB.

Compared with the normal controls, the patients who accepted early surgical treatment presented with increased MUSIX values in both $\mathrm{AH}$ and $\mathrm{EDB}$ on right side $(P<0.05$, Table 3$)$. Furthermore, the patients undergoing delayed surgical treatment showed increased MUSIX values in bilateral $\mathrm{AH}$, as well as both reduced MUNIX values and increased MUSIX values in both TA and EDB on bilateral side $(P<$ 0.05 , Table 3$)$. In addition, delayed surgical treatment 


\section{Normal subjects}
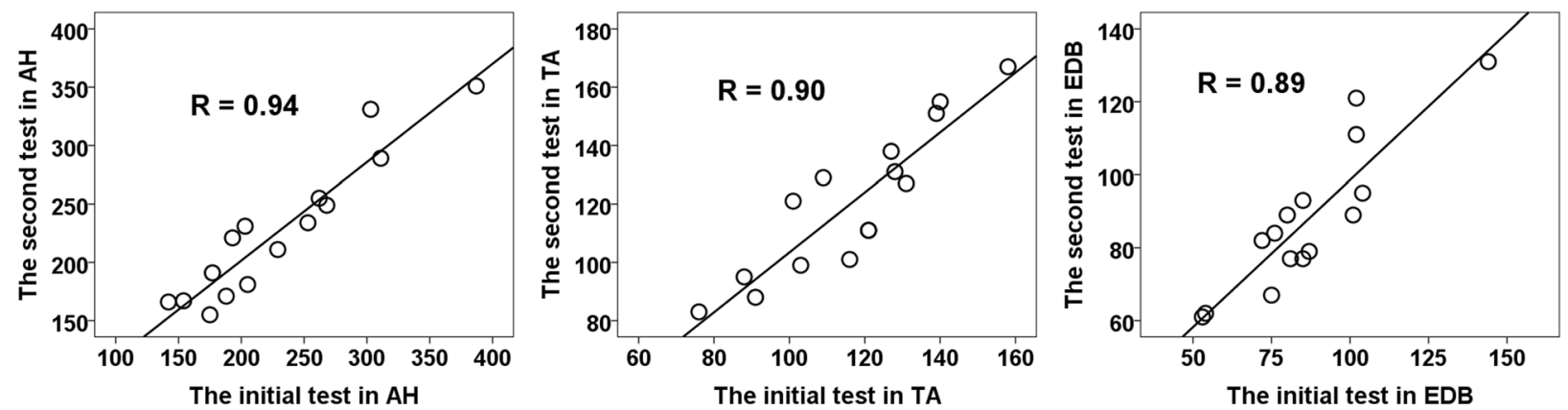

\section{Patients with cervical SCI}
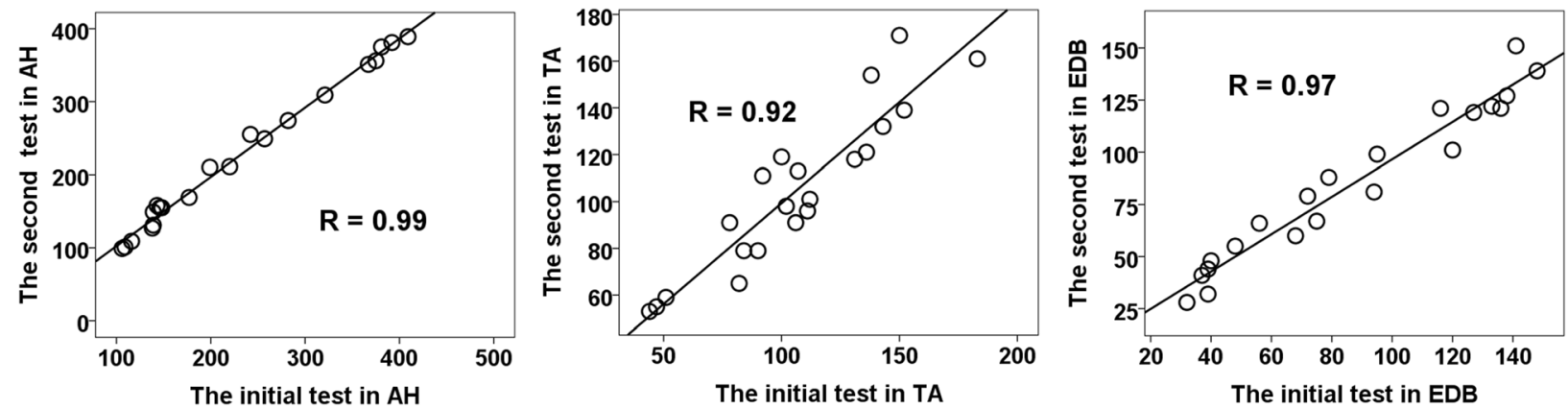

Fig. 1 Correlations between MUNIX values of the initial and the second tests in both patients with cervical SCI (left side) and healthy subjects (left side). The graphs show that there is a strong positive correlation between the values of first and the second tests in all tested muscles in both cervical SCI patient and control groups. SCI: Spinal cord injury; MUNIX: motor unit number index; AH: abductor hallucis; EDB: extensor digitorum brevis; TA: tibialis anterior

Table 2 Test-retest reproducibility of MUNIX in patients with $\mathrm{SCl}$ and healthy subjects

\begin{tabular}{lll}
\hline & Patients with cervical SCl & Healthy subjects \\
\hline Number of cases & 21 & 15 \\
Age range (years) & $49.2 \pm 11.8$ & $45.0 \pm 11.5$ \\
Height range (cm) & $163.6 \pm 9.2 \quad$ CCA & ICC CCA
\end{tabular}

Abductor hallucis

$\begin{array}{lllll}\text { CMAP } & 0.99 & 0.99 & 0.99 & 0.99 \\ \text { MUNIX } & 0.99 & 0.99 & 0.97 & 0.94 \\ \text { MUSIX } & 0.96 & 0.93 & 0.96 & 0.95\end{array}$

\section{Extensor digitorum brevis}

$\begin{array}{lllll}\text { CMAP } & 0.99 & 0.97 & 0.98 & 0.97 \\ \text { MUNIX } & 0.99 & 0.97 & 0.94 & 0.89 \\ \text { MUSIX } & 0.95 & 0.90 & 0.91 & 0.84\end{array}$

Tibialis anterior

\begin{tabular}{lllll} 
CMAP & 0.99 & 0.98 & 0.97 & 0.94 \\
MUNIX & 0.96 & 0.92 & 0.94 & 0.90 \\
MUSIX & 0.90 & 0.84 & 0.87 & 0.78 \\
\hline
\end{tabular}

MUNIX Motor unit number index, SCI Spinal cord injury, CMAP Compound muscle action potential, MUSIX Motor unit size index, ICC Intraclass correlation coefficient, CCA Correlation coefficient analysis group patients also presented with reduced CMAP amplitudes in left-side EDB $(P<0.05$, Table 3$)$.

There is no difference of ASIA motor scores between the patients who accepted early and delayed surgical treatment, and MRC scales in all tested muscles were similar between the early and delayed surgical treatment groups $(P>0.05$, Table 3$)$. In contrast, the patients undergoing early surgical treatment showed lower MUSIX values in both bilateral EDB and bilateral TA, along with greater MUNIX values in both right-side EDB and right-side TA, compared to the patients who accepted delayed surgical treatment $(P<0.05$, Table 3$)$.

\section{Discussion}

The MUNIX method used in this study only requires a few numbers of electrical stimulation, and it usually takes approximately $30 \mathrm{~min}$ to measure six muscles in one subject, thus facilitating patient compliance. More importantly, according to the results of both ICC and CCA, we identified the excellent levels of reproducibility of MUNIX measurements in both the control and SCI patient groups. Therefore, the findings of this study are technically sound and reflect reliably functional motor units of the tested muscles. 


\section{Delayed surgical treatment group}
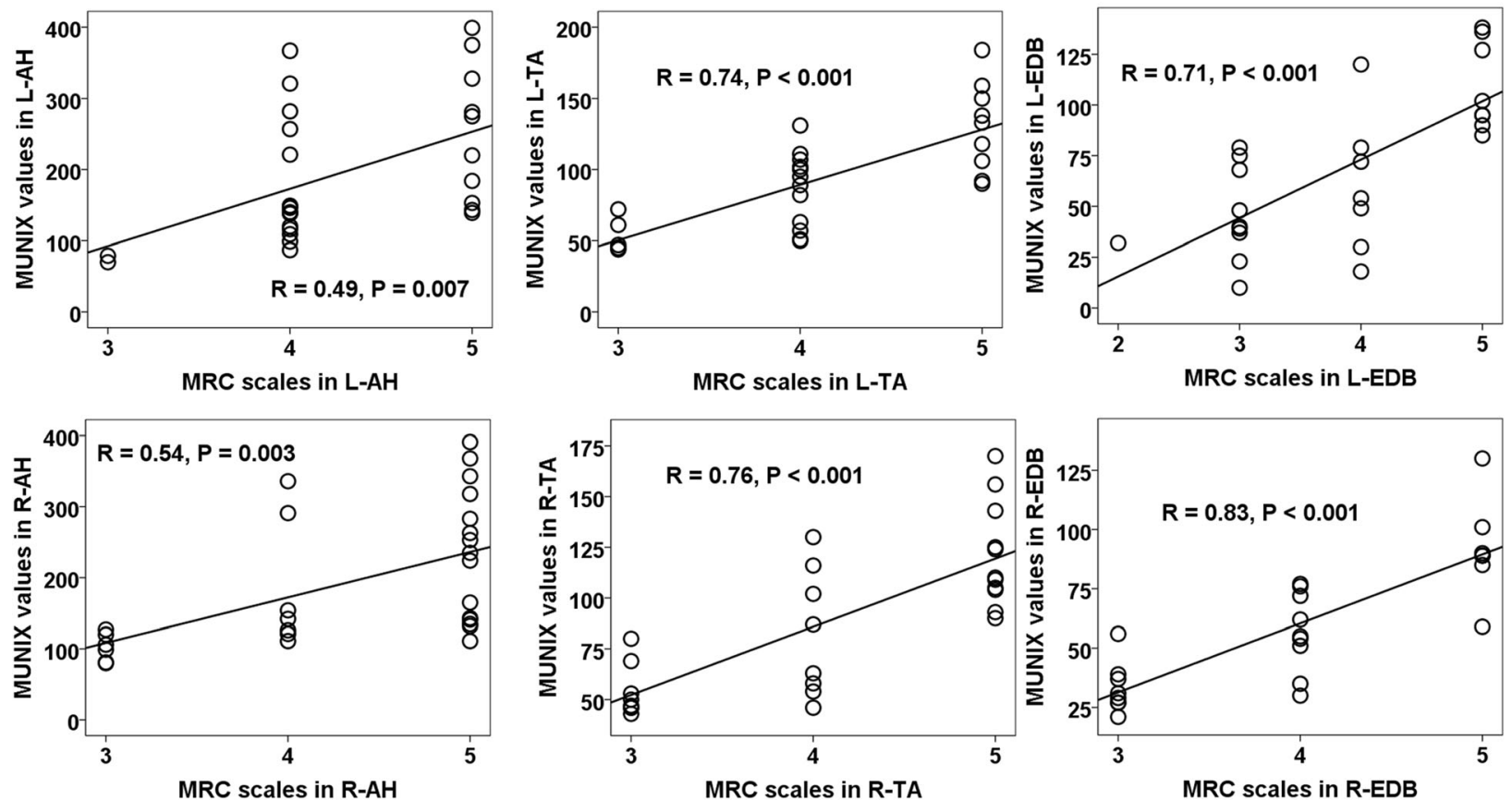

\section{Early surgical treatment group}
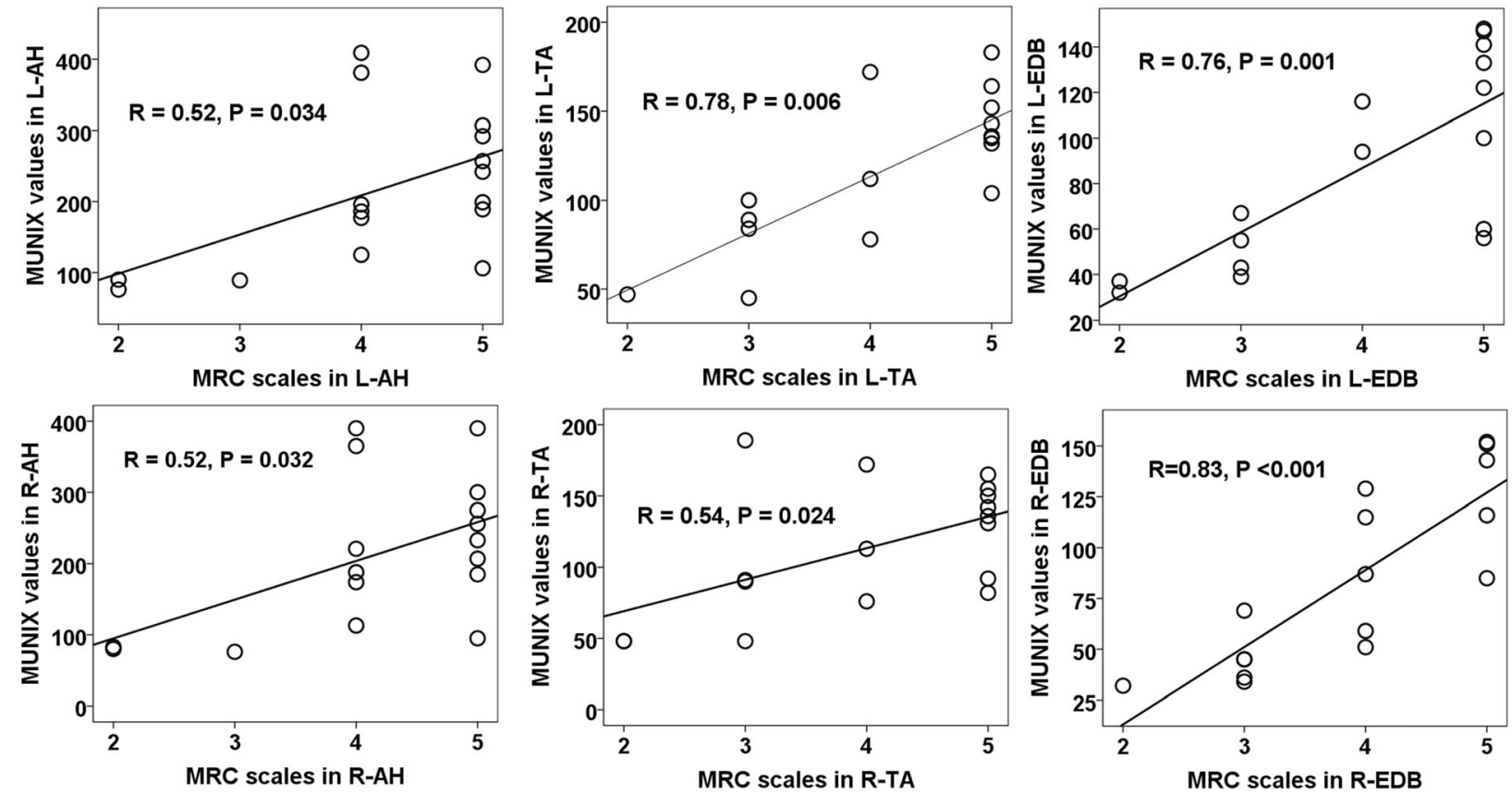

Fig. 2 Correlations between MRC scales and MUNIX values in both cervical SCI patient groups. There was a significant relationship between the MRC scales and MUNIX values in all tested muscles in both cervical SCI patient groups. SCI: Spinal cord injury; MRC: medical research council; MUNIX: motor unit number index; AH: abductor hallucis; EDB: extensor digitorum brevis; TA: tibialis anterior; L: left side; R: right side

In the present study, significant loss of motor units in the lumbosacral-innervated muscles in patients with cervical SCI, suggesting that peripheral nerve function is compromised following upper motor neuron lesion.
Central disconnection of the second-order motor neurons is considered to be the likely reason for this condition, and many previous studies have provided evidence with regard to this disconnection may cause loss of 
Table 3 Measurements of MUNIX detection and clinical function measures in both SCl patient and normal control groups

\begin{tabular}{|c|c|c|c|c|c|c|}
\hline & \multicolumn{2}{|c|}{ Early surgical treatment group } & \multicolumn{2}{|c|}{ Delayed surgical treatment group } & \multicolumn{2}{|c|}{ Healthy subjects } \\
\hline Number of subjects & \multicolumn{2}{|l|}{17} & \multicolumn{2}{|l|}{30} & \multicolumn{2}{|l|}{34} \\
\hline Age range (years) & \multicolumn{2}{|l|}{$45.0 \pm 12.2$} & \multicolumn{2}{|l|}{$47.4 \pm 13.1$} & \multicolumn{2}{|l|}{$44.0 \pm 11.1$} \\
\hline \multirow[t]{2}{*}{ Height range $(\mathrm{cm})$} & \multicolumn{2}{|l|}{$164.7 \pm 8.9$} & \multicolumn{2}{|l|}{$165.9 \pm 9.2$} & \multicolumn{2}{|l|}{$166.9 \pm 5.7$} \\
\hline & Left side & Right side & Left side & Right side & Left side & Right side \\
\hline \multicolumn{7}{|l|}{ MUNIX detection } \\
\hline CMAP-AH & $14.0 \pm 4.7$ & $14.1 \pm 4.8$ & $13.4 \pm 4.7$ & $13.3 \pm 4.5$ & $14.6 \pm 4.9$ & $14.2 \pm 5.0$ \\
\hline MUNIX-AH & $218.4 \pm 108.3$ & $213.6 \pm 106.0$ & $195.9 \pm 97.5$ & $192.8 \pm 96.0$ & $240.8 \pm 96.8$ & $240.4 \pm 103.5$ \\
\hline MUSIX-AH & $71.5 \pm 20.3$ & $74.8 \pm 24.2^{*}$ & $76.8 \pm 14.1^{*}$ & $77.1 \pm 14.9^{*}$ & $63.0 \pm 12.5$ & $62.7 \pm 13.7$ \\
\hline CMAP-TA & $6.1 \pm 1.3$ & $6.1 \pm 1.1$ & $5.9 \pm 1.8$ & $5.9 \pm 1.5$ & $6.5 \pm 1.0$ & $6.5 \pm 1.0$ \\
\hline MUNIX-TA & $117.3 \pm 41.9$ & $113.4 \pm 45.6 \#$ & $95.3 \pm 38.2^{*}$ & $89.6 \pm 37.2^{*} \#$ & $122.9 \pm 31.7$ & $119.6 \pm 30.8$ \\
\hline MUSIX-TA & $58.1 \pm 20.3 \#$ & $61.1 \pm 22.2 \#$ & $70.6 \pm 15.6^{*} \#$ & $75.7 \pm 20.5^{*} \#$ & $55.2 \pm 10.4$ & $56.4 \pm 11.3$ \\
\hline CMAP-EDB & $5.6 \pm 2.2$ & $5.6 \pm 2.3$ & $5.0 \pm 2.0^{*}$ & $5.0 \pm 2.0$ & $6.0 \pm 1.5$ & $5.9 \pm 1.9$ \\
\hline MUNIX-EDB & $86.9 \pm 42.8$ & $84.3 \pm 44.0 \#$ & $69.6 \pm 37.4^{*}$ & $60.4 \pm 28.5^{*} \#$ & $94.8 \pm 30.1$ & $94.7 \pm 34.4$ \\
\hline MUSIX-EDB & $73.8 \pm 19.2 \#$ & $76.7 \pm 18.3^{*} \#$ & $87.7 \pm 30.2^{*} \#$ & $92.9 \pm 20.0^{*} \#$ & $65.2 \pm 13.6$ & $63.7 \pm 12.2$ \\
\hline \multicolumn{7}{|c|}{ Clinical function measures } \\
\hline MRC-AH & $4.2 \pm 1.0$ & $4.2 \pm 1.0$ & $4.1 \pm 0.8$ & $4.2 \pm 1.0$ & \multirow[t]{2}{*}{ / } & \multirow[t]{2}{*}{ / } \\
\hline MRC-TA & $3.9 \pm 1.2$ & $4.0 \pm 1.1$ & $3.9 \pm 1.0$ & $3.9 \pm 1.0$ & & \\
\hline MRC-EDB & $3.8 \pm 1.3$ & $3.7 \pm 1.2$ & $3.7 \pm 1.0$ & $3.7 \pm 1.0$ & / & / \\
\hline ASIA motor score & \multicolumn{2}{|l|}{$81.4 \pm 15.2$} & \multicolumn{2}{|l|}{$78.6 \pm 18.3$} & \multicolumn{2}{|l|}{ l } \\
\hline
\end{tabular}

SCI Spinal cord injury, MUNIX Motor unit number index, CMAP Compound muscle action potential, MUSIX Motor unit size index, TA tibialis anterior, AH abductor hallucis, EDB Extensor digitorum brevis, MRC Medical research council score, ASIA American spinal injury association

* Statistical difference between the patient and control groups, $P<0.05$

\# Statistical difference between the early and delayed surgical treatment groups, $P<0.05$

lower motor neurons (LMNs) distal to the initial injury site, which may cause the motor axonal degeneration and an eventual reduction in motor units $[1,5,12-14]$.

Although the measurements of MUNIX detection were obvious difference between the patients who accepted early and delayed surgical intervention approximately 1 year after operation, both CMAP amplitudes in both $\mathrm{AH}$ and TA and MRC scores in all tested muscles were similar between these two patient groups. This condition may be ascribed to these evaluation methods are relatively suboptimal to detect subtle difference between different treatments. Similar results were also reported in many previous studies [8, 11, 15-17], and collateral sprouting induced by longstanding neurodegeneration is likely the main reason. The remarkable increase in MUSIX values in the tested muscles in this study further identified the existence of the reinnervation from the surviving motor axons. According to previous studies, the reinnervation process can provide a functional compensatory mechanism to preserve muscle strength, and recently published study further indicated muscle strength usually can be preserved by reinnervation until $50 \%$ or more of motor units are lost $[1,18$, 19]. Therefore, compared to current clinical biomarkers (e.g., CMAP amplitudes and muscle strength), MUNIX may be a more objective, sensitive and reliable method for monitoring the treatment outcomes of SCI. Although clinical function improvement is very important to the patients with SCI, the difference of MUNIX measurements between two different treatment group may provide additional unique information for guiding the doctors to select treatment modalities.

When reviewing these findings, one of the limitations is that MUNIX detection is easily influenced by both the examiner and the protocol (e.g., recording of maximum CMAP amplitude). Thus, a standard protocol and a fixed examiner were used in the current study [8]. Furthermore, another limitation is that the time cutoffs by which to identify early vs. delayed surgical treatment were different in various studies involving SCI (e.g., $12 \mathrm{~h}, 24 \mathrm{~h}, 4$ days and 2 weeks) $[20,21]$. Although the definition of early surgery as within the first 2 weeks is not the most common time cutoff, the recent systematic review demonstrated it is preferable to operate within 2 weeks compared to operation within $24 \mathrm{~h}$ [22]. Furthermore, few patients can accept surgical treatment within $24 \mathrm{~h}$, due to both transportation issues and referral delays. The other clinical limitation of this study is low sample size, which may be ascribed to the fewer patients with incomplete cervical SCI have complete medical records and more patients lost to follow-up. More significant results might be achieved in future studies with an increased number of both cases and sub-groups. 


\section{Conclusion}

Cervical SCI has a potential negative effect on the motor neurons distal to the level of injury. Equally important, different follow-up results of the MUNIX detection between the early vs. delayed surgical treatment group supported that early surgical intervention in some patients with SCI may relatively improve the dysfunction of LMNs and reduce the loss of motor neurons, eventually improving clinical prognosis.

\begin{abstract}
Abbreviations
SCI: Spinal cord injury; UMN: Upper motor neuron; LMN: Lower motor neuron; MUNIX: Motor unit number index; TA: Tibialis anterior; EDB: Extensor digitorum brevis; AH: Abductor hallucis; CMAP: Compound muscle action potential; SIP: Surface interference pattern; MUSIX: Motor unit size index; MRC: Medical Research Council; ASIA: American spinal injury association; CCA: Correlation coefficient analysis; ICC: Interclass correlation coefficient
\end{abstract}

\section{Acknowledgements}

Not applicable.

\section{Authors' contributions}

HE S and WANG D have made substantial contributions to conception and design; LI J and ZHU Y have made substantial contributions to acquisition of data, or analysis and interpretation of data; LI Y have been involved in drafting the manuscript or revising it critically for important intellectual content; all authors have given final approval of the version to be published.

\section{Funding}

All authors (HE S, WANG D, LI J, ZHU Y and LI Y) declare that Financial support from the Foundation of science and Technology Commission of Songjiang District, Shanghai (18sjgg9931).

\section{Availability of data and materials}

The datasets used and/or analysed during the current study are available from the corresponding author on reasonable request.

\section{Ethics approval and consent to participate}

All authors (HE S, WANG D, LI J, ZHU Y and LI Y) declare that the study protocol was approved by Human Ethics Committees (Shanghai Songjiang District Central Hospital). All subjects gave informed consent through written.

\section{Consent for publication}

Not applicable.

\section{Competing interests}

All authors (HE S, WANG D, LI J, ZHU Y and LI Y) declare that they have no competing interests.

\section{Author details}

${ }^{1}$ Department of Orthopedics, College of Clinical Medicine, Shanghai Ten Hospitals of Nanjing Medical University, 301 Yanchang Middle Road, Jing'an District, Shanghai 200072, China. ${ }^{2}$ Department of Orthopedics, Shanghai Songjiang District Central Hospital, Shanghai 201600, China. ${ }^{3}$ Department of Orthopedics, The Second Affiliated Hospital of Nanjing Medical University, Nanjing 210011, Jiangsu Province, China.

Received: 13 March 2020 Accepted: 3 August 2020

Published online: 15 August 2020

\section{References}

1. Van De Meent H, Hosman AJ, Hendriks J, et al. Severe degeneration of peripheral motor axons after spinal cord injury: a European multicenter study in 345 patients. Neurorehabil Neural Repair. 2010;24:657-65.

2. Yelamarthy PKK, Chhabra HS, Vaccaro A, et al. Management and prognosis of acute traumatic cervical central cord syndrome: systematic review and spinal cord society-spine trauma study group position statement. Eur Spine J. 2019;28:2390-407.
3. Divi SN, Schroeder GD, Mangan JJ, et al. Management of Acute Traumatic Central Cord Syndrome: a narrative review. Global Spine J. 2019;9:89S-97S

4. Lenehan B, Fisher CG, Vaccaro A, et al. The urgency of surgical decompression in acute central cord injuries with spondylosis and without instability. Spine (Phila Pa 1976). 2010;35:S180-6.

5. Zheng C, Zhu Y, Lu F, et al. Trans-synaptic degeneration of motoneurons distal to chronic cervical spinal cord compression in cervical spondylotic myelopathy. Int J Neurosci. 2017;127:988-95.

6. Zheng C, Nie C, Zhu Y, et al. Changes in central motor conduction time and its implication on dysfunction of distal upper limb in distal-type cervical Spondylotic Amyotrophy. J Clin Neurophysiol. 2019;36:52-9.

7. Xiong GX, Zhang JW, Hong Y, et al. Motor unit number estimation of the tibialis anterior muscle in spinal cord injury. Spinal Cord. 2008;46:696-702.

8. Nandedkar SD, Barkhaus PE, Stålberg EV. Motor unit number index (MUNIX): principle, method, and findings in healthy subjects and in patients with motor neuron disease. Muscle Nerve. 2010;42:798-807.

9. Philibert M, Grapperon A, Delmont E, et al. Monitoring the short-term effect of intravenous immunoglobulins in multifocal motor neuropathy using motor unit number index. Clin Neurophysiol. 2017;128:235-40.

10. Zheng $C$, Chen Z, Zhu Y, et al. Motor unit number index in quantitatively assessing motor root lesions and monitoring treatment outcomes in patients with lumbosacral radiculopathy. Muscle Nerve. 2020;61:759-66.

11. Zheng C, Song J, Zhu Y, et al. Motor unit number index (MUNIX) in the quantitative assessment of severity and surgical outcome in cervical spondylotic amyotrophy. Clin Neurophysiol. 2019;130:1465-73.

12. Mandeville RM, Brown JM, Sheean GL. A neurophysiological approach to nerve transfer to restore upper limb function in cervical spinal cord injury. Neurosurg Focus. 2017;43:E6.

13. Li X, Jahanmiri-Nezhad F, Rymer WZ, et al. An examination of the motor unit number index (MUNIX) in muscles paralyzed by spinal cord injury. IEEE Trans Inf Technol Biomed. 2012;16:1143-9.

14. Zheng C, Yu Q, Shan X, et al. Early surgical decompression ameliorates dysfunction of spinal motor neuron in patients with acute traumatic central cord syndrome: an Ambispective cohort analysis. Spine. 2020. https://doi. org/10.1016/j.wneu.2020.01.171.

15. Escorcio-Bezerra ML, Abrahao A, de Castro I, et al. MUNIX: reproducibility and clinical correlations in amyotrophic lateral sclerosis. Clin Neurophysiol. 2016;127:2979-84

16. Querin $\mathrm{G}$, Lenglet $\mathrm{T}$, Debs $\mathrm{R}$, et al. The motor unit number index (MUNIX) profile of patients with adult spinal muscular atrophy. Clin Neurophysiol. 2018;129:2333-40

17. Bas J, Delmont E, Fatehi F, et al. Motor unit number index correlates with disability in Charcot-Marie-tooth disease. Clin Neurophysiol. 2018;129:1390-6.

18. Fukada $\mathrm{K}$, Matsui $\mathrm{T}$, Furuta $\mathrm{M}$, et al. The motor unit number index of subclinical abnormality in amyotrophic lateral sclerosis. J Clin Neurophysiol. 2016:33:564-8.

19. Zheng C, Zhu Y, Zhu D, et al. Motor unit number estimation in the quantitative assessment of severity and progression of motor unit loss in Hirayama disease. Clin Neurophysiol. 2017;128:1008-14.

20. Aarabi B, Akhtar-Danesh N, Chryssikos T, et al. Efficacy of ultra-early ( $<12$ h), early (12-24 h), and late (>24-138.5 h) surgery with magnetic resonance imaging-confirmed decompression in American spinal injury association impairment scale grades a, B, and C cervical spinal cord injury. J Neurotrauma. 2020;37:448-57.

21. Wilson JR, Witiw CD, Badhiwala J, et al. Early surgery for traumatic spinal cord injury: where are we now? Global Spine J. 2020;10:84S-91S.

22. Anderson KK, Tetreault L, Shamji MF, et al. Optimal timing of surgical decompression for acute traumatic central cord syndrome: a systematic review of the literature. Neurosurgery. 2015;77:S15-32.

\section{Publisher's Note}

Springer Nature remains neutral with regard to jurisdictional claims in published maps and institutional affiliations. 